\title{
THE STEADY STATE PROPAGATION OF A DAMAGED ZONE OF AN ELASTIC BRITTLE SOLID
}

H. D. Bui*, A. Ehrlacher** and C. Renard*** Electricité de France and Ecole Polytechnique

Electricité de France and Ecole Polyt
$*$ "Ecole Polytechnique and Ecole Nationale des
"*n" Ecole Potytechnique

ABSTRACT

An aproximate solution is given for studying the steady state propagation of the damaged zone in an elastic brittle solid in plane strain. Use is made of the Muskhelishvili method of conformal madoing.

KEYWORDS

Elastic brittle, Damaged zone, Conformal mapping.

INTRODUCTION

The most simple model of damage is the elastic brittle material.

In that material the parameter of damage D introduced by Kachanov and Lemaitre [1], [2], [3] can only take two separate va-
lues:. D $=0$ as long as a critical strain

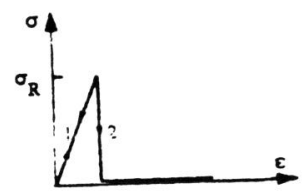
lues: $D=O$ as long as a critical strain $\varepsilon_{R}$ has not been exce

$$
|\underline{\underline{\varepsilon}}| \leqslant \varepsilon_{R}
$$

$\mathrm{D}=1$ if the critical strain has been exceeded. Then the material is totally damaged and the stress has dropped to

Fig. 1. Elastic-brittle material zero (fig. 1) :

$$
\underline{\underline{\sigma}}=0
$$

An elastic brittle solid $\Omega$ is divided into two zones :

the first one in which $D=0$ is the undamaged zone $\Omega(t)$

. the second one in which $D=1$ is the damaged zone $\Omega_{d}(t)$. The damaged zone grows with time.

The boundary between the undamaged zone and the damaged zone is divided into the 
- on the second one the critical strain is reached

$$
|\varepsilon|=\varepsilon_{R}
$$

A part of the boundary moves toward the undamaged zone when the damaged zone is growing. We call it the damage front

That boundary is a free boundary because $\sigma=0$ in the damaged zone.

Some problems dealing with thus catastrophic damage model have been already studied : - dynamic steady state propagation of the damaged zone in antiplane mode. The shape of the damage front is then a cycloidd [4], $[5]$

- the equation of the normal speed of the damage front in non steady state propagation of the damaged zone in antiplane mode has been established in [6]; it is a Prandt1's equation - a variational formulation for the field of rate of stress and

Here we shall study the steady state propagation of the damaged zone in plane strain.

STEADY STATE PROPAGATION OF THE DAMAGED ZONE IN PLANE STRAIN Let us consider a plane, infinite, elastic brittle solid in plane strain with a damaged zone $\Omega_{d}(t)$ propagating through the solid at speed $v$. The steady state regime is established (fig.2).

If we look at the damage zone in the moving coordinates axes $\left(0, x_{1}, x_{2}\right)$ propagating at the same speed $\mathrm{V}$, the boundary of the damaged zone seems fixed, and is divided into three parts :

- two straight lines $A_{4} A_{3}$ and $A_{1} A_{2}$ on which the critical strain is not rea-

- the damage front $\mathrm{A}_{3} \mathrm{~A}_{2}$.

Our problem will be to determine the shape of that front $A_{3} A_{2}$.

On the undamaged side of the damage front the strain tensor can be expressed by means of the unique scalar value of the tangential traction stress $\sigma_{t t}$ :

$$
\sigma_{t t}=\underline{t} \cdot \underline{\sigma} \cdot \underline{t}
$$

where $t$ is the unit vector tangent to the front and instead of (3) we can write :

$$
\sigma_{t t}=\sigma_{R} \quad \text { on } A_{3} A_{2}
$$

where $\sigma_{R}$ is a material constant depending on $\varepsilon_{R}$.

If we look at our damage zone from a great distance (infinity), it looks like a crack, so we take the same fields at infinity as for the problem of the semi-infinite crack, but the parameter $K_{\mathrm{I}}$ which is a stress intensity infinity.
inf crack problem is in our problem only a loading parameter at

Using an invariant integral it has been established in [5] a relation between the half width $h$ of the damaged zone, the loading parameter $\mathrm{K}_{\mathrm{I}}$ and the damage stress $\sigma_{R}:{ }_{h}=\frac{K_{I}{ }^{2}}{\sigma_{R}^{2}}$
CONFORMAL MAPPINGS OF THE UNDAMAGED ZONE

We consider $\omega(\xi)$, the function which maps the interior of the unit circle $|\xi|<1$ of the plane $\xi$ into the undamaged zone $\Omega_{s}$ in the $z$ plane (fig.2)$$
z=x_{1}+i x_{2}=\omega(\xi)
$$

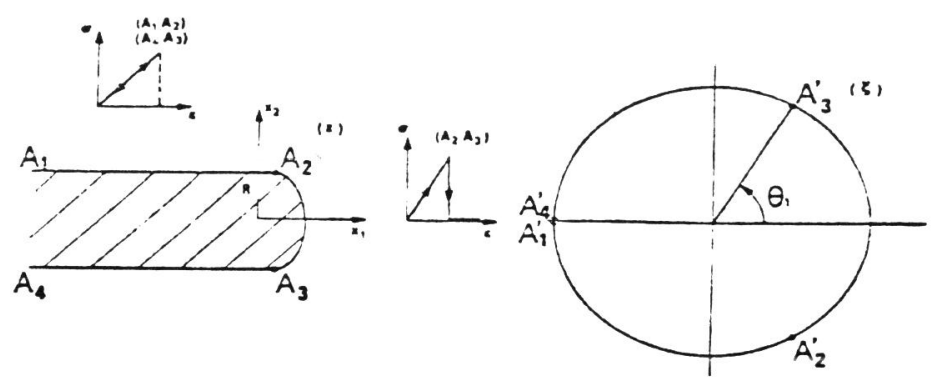

.Fig. 2. Conformal mapping of the undamaged zone

The point $\xi=-1$ gives the point at infinity in the $z$ plane and is a pole of order 2 of $\omega(\xi)$. If we can find the unknown function $\omega(\xi)$, we shall have the shape of the damage front.

We shall use the Kolosov-Muskhelishvili method [8].

The elastic solution in the undamaged zone is given by means of two complex functions $\varphi(\xi)$ and $\psi(\xi)$ which must be deduced from the boundary condition.

In particular it is well known that :

$$
\sigma_{x x}+\sigma_{y y}=4 \operatorname{Re}\left(\varphi_{(\xi)}^{\prime} / \omega^{\prime}{ }_{(\xi)}\right)
$$

and the condition $\underline{\underline{\sigma}} \cdot \underline{n}=0$ on the boundary of the undamaged zone is equivalent to :

$$
\varphi_{(\xi)}+\frac{\omega^{\prime}(\xi)}{\omega_{(\xi)}^{\prime}} \overline{\varphi^{\prime}(\xi)}+\overline{\psi(\xi)}=0 \quad \text { if }|\xi|=1
$$

The condition of loading at infinity is equivalent to :

$$
\varphi_{(z)} \approx K_{I} \sqrt{\frac{z}{2 \pi}} \quad|z| \rightarrow \infty
$$

or :

$$
\varphi_{(\xi)} \approx \mathrm{K}_{\mathrm{I}} \sqrt{\frac{\omega(\xi)}{2 \pi}} \quad \xi \rightarrow-1
$$

The damage front $A_{2} A_{3}$ in the $z$ plane corresponds to the $\operatorname{arc} A_{2}^{\prime} A_{3}^{\prime}$ in the $\xi$ plane. On that arc we have by means of (5).

$$
\sigma_{x x}+\sigma_{y y}=\sigma_{n n}+\sigma_{t t}=\sigma_{R}
$$


because $\sigma_{\mathrm{nn}}=0$, and we deduce :

$$
\operatorname{Re}\left(\varphi^{\prime}{ }_{(\xi)} / \omega_{(\xi)}\right)=\sigma_{R^{\prime}} / 4 \quad \text { on } A_{2}^{\prime} A_{3}^{\prime} .
$$

Our problem becomes : find the three functions $\omega_{(\xi)}, \varphi_{(\xi)}, \psi_{(\xi)}$ with the boundary conditions (9), (11), (13) and (14):

$$
\begin{cases}\operatorname{Im} \omega_{(\xi)}=h & \text { on } A_{1}^{\prime} A_{2}^{\prime} \\ \operatorname{Im} \omega_{(\xi)}=-h & \text { on } A_{3}^{\prime} A_{4}^{\prime}\end{cases}
$$

and with $\omega(\xi)$ having a pole of order two at $\xi=-1$.

This is a non-linear problem. In the next section we shall look for an approximate solution.

APPROXIMATE SOLUTION

We shall write $\omega(\xi)$ as :

$$
\omega(\xi)=\left(\sum_{0}^{\infty} \mathrm{T}_{k} \xi^{\mathrm{k}}\right) /(1+\xi)^{2}
$$

$\left(\omega(\xi)(1+\xi)^{2}\right.$ is holomorphic in the unit circle and can be expanded in a series).

This transformation has already been used by V.D. Kuliev [9] to study the problem of a semi-infinite rectangular cutout. The $T_{k}$ are unknown and must be found from conditions on the damage front.

We shall have an approximate shape if we cruncate the series

$$
\omega_{N}(\xi)=\left(\sum_{0}^{N} T_{k} \xi^{k}\right) /(1+\xi)^{2} \quad \text { (16) }
$$

To have a finite width of the damaged zone at infinity we must have :

$$
\sum_{0}^{\infty}(-1)^{\mathrm{k}} \mathrm{T}_{\mathrm{k}}(\mathrm{k}-1)=0
$$

For each value of $\mathrm{N}$ we can have the exact elastic solution of the approximate problem.

Let $\varphi_{N}$ and $\psi_{\mathrm{N}}$ be the two functions which give the elastic solution with the approximate shape of the undamaged zone deduced from $\omega_{N}$. We can write :

$$
\left\{\begin{array}{l}
\varphi_{N}(\xi)=\sum_{0}^{\infty} a_{k} \xi^{h} /(1+\xi) \\
\psi_{N}(\xi)=\sum_{1}^{\infty} c_{k} \xi^{h} /(1+\xi)
\end{array}\right.
$$
The use of (9) and (11) allows us to calculate explicitly the $a_{k}$ and $c_{k}$ by means of a
to $[9],[10]$.

As we are searching for an approximate solution, we must define the error $\Delta\left(\omega_{N}\right)$ between the approximation and the exact solution.

The error $\Delta\left(\omega_{N}\right)$ will be divided into two parts : the first

$$
\Delta_{1}\left(\omega_{N}\right)=\int_{0}^{\theta_{1}}\left(\operatorname{Re} \frac{\varphi_{N}^{\prime}}{\omega N}-\frac{\sigma_{R}}{4}\right)^{2} d \theta
$$

the second one measures the error on the geometry :

$$
\Delta_{2}\left(\omega_{N}\right)=\int_{\theta_{1}}^{\theta}\left(\operatorname{Im} \omega_{N}-h\right)^{2} d \theta
$$

We choose the total error as :

$$
\Delta\left(\omega_{N}\right)=\lambda \Delta_{1}\left(\omega_{N}\right)+\mu \Delta_{2}\left(\omega_{N}\right)
$$

In (19) $\theta_{1}$ is the unknown parameter of the end of the damage front. A mini misation process leads us to choose $\theta_{1}=0.965$ radians, but the influence of that parameter is weak since different values between 0.93 and 0.98 give similar results.

In (20) $\theta_{2}$ is a parameter between $\theta_{1}$ and $\pi$ which is related to the distance from the 2 ls a pant on which we want to have a good approximation to the straight line $A_{1} A_{2}$. We have choosen arbitrarily

$$
\theta_{2}=\frac{2 \pi}{3}
$$

which gives a good approximation far enough from the damage front.

In (21) parameters $\lambda$ and $\mu$ in such a way that $\lambda \Delta_{1}$ and $\mu \Delta_{2}$ In

Now our error is completely defined by (21) and $\Delta\left(\omega_{N}\right)$ is a function of the $\mathrm{N}+1$ scalar unknowns $\left(\mathrm{T}_{k}\right)$. We shall say that the better approximation at order $\mathrm{N}$ is given by the $\left(\mathrm{T}_{k}\right)$ which minimise $\Delta\left(\omega_{N}\right)$.

That minimisation can be done quite easily with a computer and we obtain a better approximation when we increase $\mathrm{N}$.

\section{APPROXIMATE SOLUTION OBTAINED AFTER MINIMISATION}

We have calculate the parameters $T_{k}$ which minimise the error $\Delta \omega N_{\text {, with }} 4$, 5,11 and 20 parameters. The error then is equal to $\Delta\left(\omega_{20}\right)=1.8267 .10^{-5}$. To obtain a better approximation with more parameters would have cost too in the error.

In figure 3 we have given the shape of the approximate damaged zone obtained with the 20 parameters which minimise $\Delta\left(\omega_{20}\right)$.

We For comparison we have reproduced the solution of the same problem obtained by a finite element method in $[5]$. 


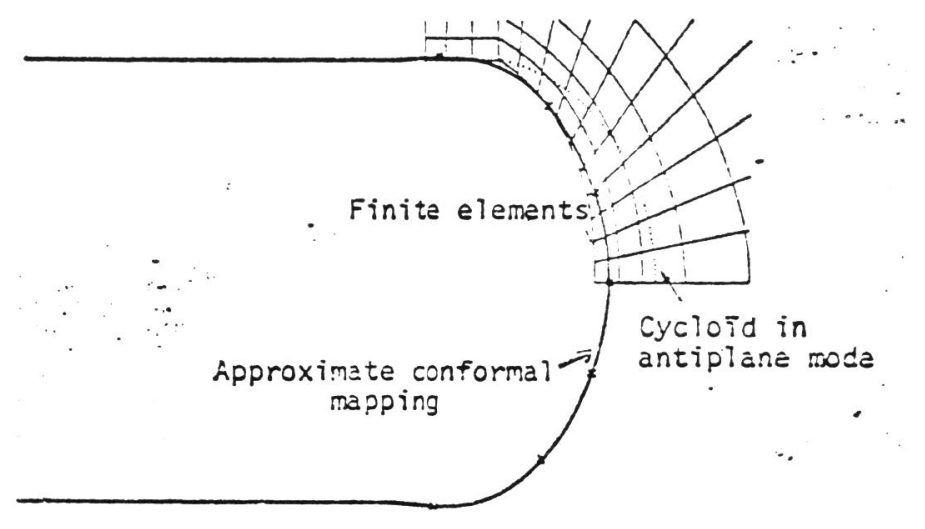

Fig. 3. Approximate solution with 20 parameters

In figure 4 we can see the error between $\sigma$ and $\sigma$ on the approximate damage front. The error is less than $1 \%$ for the greatest part of the damage front.

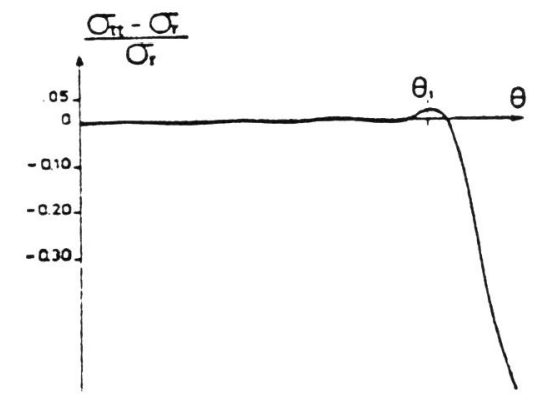

Fig. 4. Difference between $\sigma_{t t}$ and $\sigma_{R}$

\section{REFERENCES}

[ 1 ] Kachanov, L.M., (1958), Inv. Ak. N.S.S.R., 8, 26.

[ 2 ] Lemaitre, J., and Chaboche, J.L., (1978), J. Mécan. App1., 2, n³, 317.

[ 3 ] Lemaitre, J., Cordebois, J.P., and Dufailly, J., (1979), Comptes-Rendus 288 , série B, 391.

[ 4 ] Bui, H.D. and Ehrlacher, A., (1980), Comptes-Rendus, 290, série B, 273

[ 5 ] Bui, H.D. and Ehrlacher, A., (1981), Int. Conf. on Fracture, ICF 5 , Cannes.

[ 6] Ehrlacher, A., (1981), Comptes-Rendus, 293, série II, 95.

[ 7 ] Bui, H.D., Dang Van, K. and Stolz, C., (1981), Comptes-Rendus, 292, série II, 251 .
[ 8] Muskhelishvili, N.I., ( ), Some Basic Problems of the Mathematical Theory of Elasticity, Gröningen.

[ 9 ] Kuliev, V.D., ( ), P.M.M., U.S.S.R., 44, 677-680.

[10] Renard, D., (1982), Rapport de stage d'option, L.M.S., Ecole Polytechnique. 